\section{0 \\ VESICOVAGINAL FISTULA REPAIR IN A CASE OF CANCER CERVIX: A ROBOTIC ASSISTED TECHNIQUE}

1;1T Shylasree*, ${ }^{2}$ G Prakash, 'S Gupta. 'Tata Memorial Hospital, Gynecological Oncology, Mumbai, India; ${ }^{2}$ Tata Memorial Hospital, Uro-oncology, Mumbai, India

10.1136/ijgc-2021-ESG0.51

Introduction/Background* Vesicovaginal fistula (VVF) is a rare complication of simple hysterectomy, however urinary fistulas can occur in patients when cervix and surrounding tissue is distorted due to fibroids or cervical cancer

Methodology A 43 years old lady was referred to our centre with complaints of continuous urinary incontinence post-surgery. She had undergone simple hysterectomy with salpingoophrectomy for undiagnosed cervical cancer.

Clinical examination, cystoscopy and staging contrast CT scan showed $2 \mathrm{~cm}$ defect in posterior wall of urinary bladder communicating with vagina. There was no evidence of parametrial, vaginal or lymph node disease. Review histopathology confirmed squamous cell carcinoma of cervix.

$\mathrm{Da}$ Vinci $\mathrm{Xi}$ system was used with port placements at the level of umbilicus. Prior to docking, bilateral ureteric catheters along with catheter in the fistula track was placed cystoscopically. Dome of the bladder was opened to visualise fistulous track completely. Bladder and vaginal wall were identified around the fistulous margin and mobilized. Vaginal edges were sutured in transverse direction and bladder edges were sutured in longitudinal direction so that both the suture lines were perpendicular to each other to reduce tissue tension and better healing. Continuous V-lock sutures were used for both vagina and bladder repair and an omental flap was placed at the fistula site for healing and preventing adhesions. Blood loss was $200 \mathrm{ml}$. She had an indwelling bladder catheter for 2 weeks along with a prescription of bladder relaxants

Result(s)* Her postoperative period was uneventful and CT cystogram on day 14 showed no urinary leak. She was referred for further adjuvant treatment in view of incompletely treated cervical cancer and presence of few peritoneal nodules diagnosed during repair. At 6 months follow up of VVF repair, patient is continent with no urinary complains, however she has progressive disease.

Conclusion* In conclusion, Urinary fistula repair through minimal access route is feasible and allows early recovery with reduced morbidity.

\section{TRANSVERSE VERSUS MIDLINE ABDOMINOPELVIC INCISIONS: A SYSTEMATIC REVIEW}

1J Al-Majali, ${ }^{2} \mathrm{M}$ Qasem, ${ }^{1} \mathrm{~A}$ Al-Ani ${ }^{*},{ }^{1} \mathrm{~A}$ Al Shati, ${ }^{3} \mathrm{~N}$ Qasem, ${ }^{1} \mathrm{M}$ Daas, ${ }^{4} \mathrm{M}$ Alazzam. ${ }^{1}$ The University of Jordan, Amman, Jordan; ${ }^{2}$ King Hussein Medical Center, Amman, Jordan; ${ }^{3}$ Jordan University of Science and Technology, Ar-Ramtha, Jordan; ${ }^{4}$ Oxford University Hospitals, Department of Gynaecology, Oxford, UK

\subsection{6/ijgc-2021-ESG0.52}

Introduction/Background* Abdominal gynecological surgeries are conducted using three different basic incision types including midline vertical incisions, suprapubic transverse incisions (i.e. Pfannenstiel, Maylard, and Cherney), and infra/supraumbilical incisions. Choosing the type of incision in gynecological malignancies can be quite challenging and depends on a variety of factors including patient-oriented factors and surgeon preference. Each type of incision has its own risks and benefits compared to its counterparts. This presses for further assessment and comparison of the data published prior to this date.

Methodology A systematic literature search was conducted on the CENTRAL, MEDLINE and EMBASE databases using the following keywords individually and in combination: 'midline incision', 'transverse incisions', 'Pfannenstiel', 'Maylard', 'Cherney', 'gynecologic cancers', 'ovarian cancer', 'cervical cancer', 'vaginal neoplasms', and 'uterine cancer'. The studies included were the ones outlining or comparing between surgical incisions' outcomes. All review articles, editorials, video articles, and abstracts were excluded.

Result(s)* The preliminary literature search reported 232 articles, after extensive screening it was filtered down to 11 articles that were fully compliant with the eligibility criteria. Throughout the literature, the 'midline incision' was reported 10 times while a single study compared 'paramedian incision' with different transverse incisions.

Conclusion* The dominance of the vertical midline incisions over transverse incisions is in constant question. Some texts remain doubtful of the applicability of the transverse incision as a valid alternative. Other articles promote the equivalence of the transverse approach to the midline regarding access to anatomical spaces, with cosmetic superiority and lowered relative risks of clinical outcomes if utilized appropriately.

\section{9 \\ CERVICAL CANCER PREVENTION POLICY ATLAS EUROPE}

N Datta, M Davidashvili*. EPF European Parliamentary Forum on Sexual and Reproductive Rights, Brussels, Belgium

\subsection{6/ijgc-2021-ESG0.53}

Introduction/Background* The Cervical Cancer Prevention Policy Atlas is a comparative map that scores

46 countries across geographical Europe (not only the European Union) on prevention policies of cervical cancer. The Atlas compares the countries on:

1. Primary prevention of cervical cancer through HPV vaccination

2. Secondary prevention of cervical cancer through screening programs, and

3. Online information on HPV, cervical cancer and accessing vaccination

It does not reflect the prevalence rate of cervical cancer in the countries or programmatic performance. The Atlas aims is to serve as a baseline to compare policies on HPV in Europe and concretely to:

- Establish the need for HPV prevention by highlighting inequity of access.

- Educate national stakeholders on the issue

- Spark debate with key policy makers at the most appropriate levels (national, regional and international)

Methodology We scored 46 European countries based on 3 headings, 9 criteria and 14 sub-criteria using the Analytic Hierarchy Process (AHP). AHP method is about setting a general, overall goal and further breaking it down the headings, criteria and sub-criteria, resembling the 'tree and the branches'. Each final 'branch', the smallest sub-criteria has its specific weight and based on the answer will receive a 


\section{CERVICAL CANCER PREVENTION POLICY ATLAS
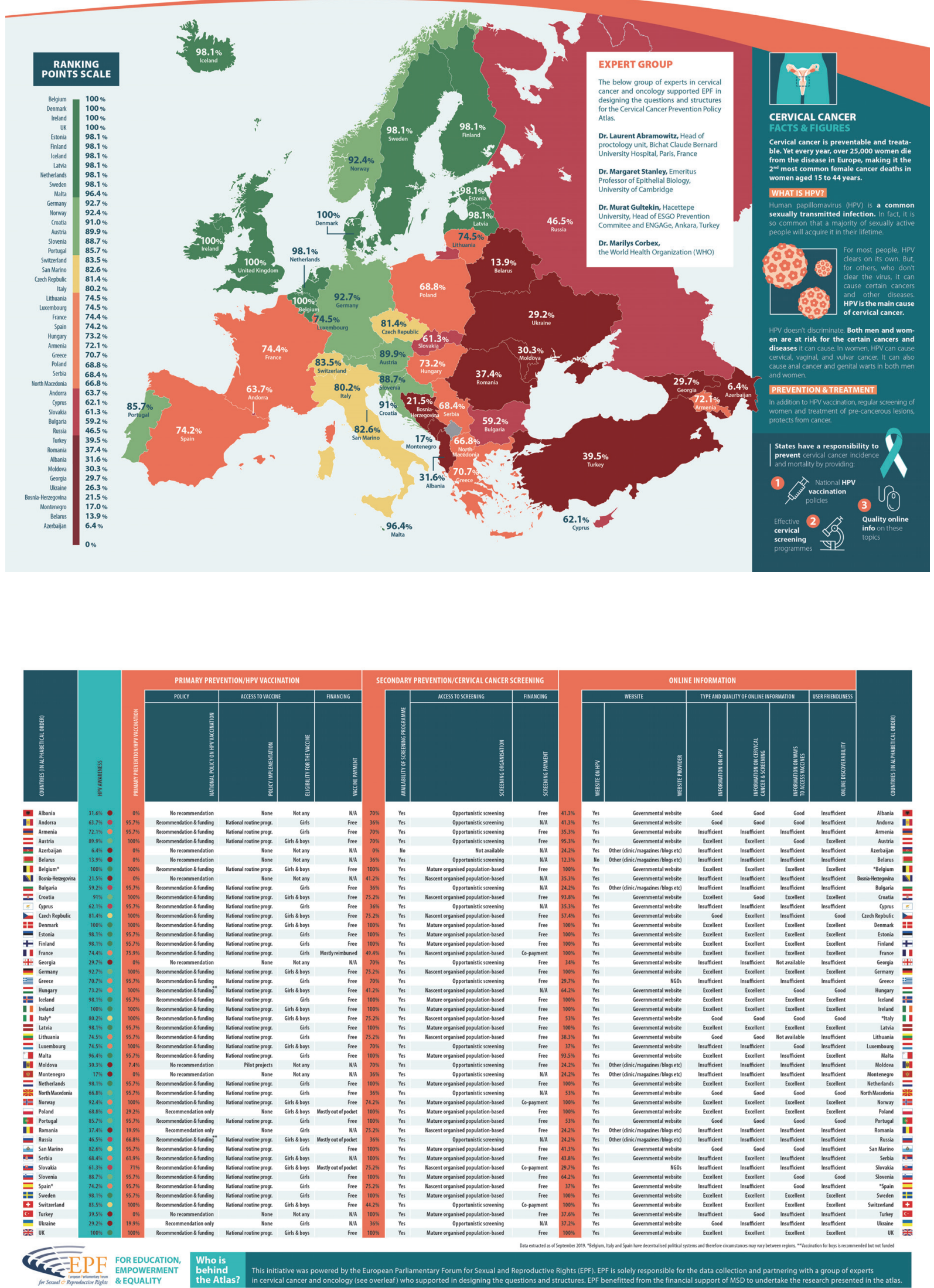
percentage score. Finally the scores for each sub-criteria are added up to the total score of each country.

Result(s)* Belgium, Denmark, Ireland and the UK are the policy champions and lead the Atlas with excellent policies on primary secondary prevention and providing evidence based information to citizens.

Romania, Bulgaria and Slovakia worst performing countries in the EU (no funding for vaccines, vaccine only available to girls, poorly organised screening programmes, absence of reliable online information). In terms of geographical Europe, Belarus and Azerbaijan score the worse, as there is literally no information about the HPV prevention to be found and policies on primary or secondary prevention are non-existent.

Conclusion* The situation in Europe is very unequal. There is a clear divide between northern Europe, Southern Europe and Eastern Europe. While vaccine exists and screenings technologies are available - today the access is very dependent on where you live. This leads to high incidence and mortality which could be avoided should proper policies be put in place.

Links ATLAS:

EPF:

\section{RAISING ADEQUATE VAGINAL MARGINS DURING COLPOTOMY FOR CERVICAL CANCER}

T Shylasree*, B Dash, P Poddar. Tata Memorial Hospital, Gynecological Oncology, Mumbai, India

\subsection{6/ijgc-2021-ESGO.54}

Introduction/Background* Adequate surgical vaginal margins are pre-requisite for improving oncological outcomes in cervical cancer and precancer. Raising the margins through vaginal route helps in visualizing and measuring the vaginal and is more accurate than performing colpotomy from a open abdominal/Minimal access route.

Methodology Following completion of ligation of uterine vessels with or without adequate parametrium depending on the indication for radicality (abdominal/minimal access route,/ schuatas vaginal hysterectomy), surgeon moves to the bottom end of the patient. Cervix is visualised and held with volsellum. Circumferential vaginal margin which needs to be removed is marked with cautery. Vaginal mucosa is infiltrated with saline with or without adrenaline. With the help of electrical diathermy vaginal margins are raised all around and separated from underlying cervix. Care should be taken not to be too close to bladder or rectum during dissection. Bladder and pouch of doughlas peritoneum is incised and uterus/cervix delivered depending on the procedure (trachelectomy/radical hysterectomy)

Result(s)* Vaginal is closed with absorbable sutures and specimen sent for final histopathology

Conclusion* Adequate Vaginal margin is a major prognostic factor in cervical cancer. Inadequate or positive margin is associated with recurrence and poor oncological outcomes, hence adjuvant postoperative radiation is indicated in such scenario. Direct visualization and measurement of vaginal to be removed and performing vaginal colpotomy ensures adequate vaginal margin and also prevents the disease being exposed to peritoneal cavity especially in minimal access surgery.

\section{THE UTERINE RADIATION NECROSIS AFTER DEFINITIVE CHEMOIRRADIATION - IMAGING AND CONTROVERSY, A SINGLE CASE REPORT}

M Mikovic*, A Tomasevic, M Radović, D Marjanovic Djoric, I Dedovic Stojakovic, V Plesinac Karapandzic. Institute of Oncology and Radiology of Serbia, Department of Radiotherapy, Belgrade, Serbia

\subsection{6/ijgc-2021-ESG0.55}

Introduction/Background* distinguishing radiation necrosis of uterus and/or cervix from central rest/recurrence after definitive chemoradiation of locally advanced cervical cancer might be challenging, even for experienced clinicians, despite various diagnostic procedures. This is a rare condition and needs to be treated with intensive local care, while central recurrence requires specific oncological treatment with a good prognosis if operable.

Methodology We present a woman, age 40 with severe acute lower abdominal pain, ten months after completing definitive chemoradiation of FIGO stage IIb cervical cancer, with an initially estimated complete treatment regression effect. Histologically, it was large cell nonkeratinizing HG2, NG2 planocellulare invasive carcinoma with a tumor-cervix diameter of $47 \mathrm{~mm}$. Total transcutaneous (TRT) dose of $46 \mathrm{~Gy}$ in 25 fractions was delivered to the whole pelvis (Rapid arc planned), with 5 cycles of weekly Cisplatin-based chemotherapy $\left(40 \mathrm{mg} / \mathrm{m}^{2}\right)$ and 5 intracavitary brachytherapy applications, 1 weekly, with a dose of 7 Gy to reference point A/per application (central tube and two ovoids). After 10 months of complete regression of cancer, clinical exam, ultrasound (US), Positron emission tomography/computed tomography (PET/CT with standardized uptake value, SUV maximum 9.5) and computed tomography (CT) showed an inhomogeneous mass of the cervix, $5 \mathrm{~cm}$ in longitudinal dimension, propagating towards rectum, strongly suspected to recurrence. A biopsy was performed with a result of necrotic inflamed tissue.

Result(s)* Due to the large scale of symptoms of inflammation, specific treatment was not conducted at the time. The patient was treated with supportive therapy, antibiotics, and intensive local care. Five months after the first symptoms, MR showed no signs of disease. The patient is scheduled for further MR control and follow-up.

Conclusion* radiation necrosis must be included in consideration if the result of the biopsy is negative even if most of the diagnostic procedures point towards central recurrent disease.

\section{ESGO QUALITY INDICATORS (QI) IN THE SURGICAL MANAGEMENT OF CERVICAL CANCER. CANARY ISLANDS MATERNAL AND CHILD UNIVERSITY HOSPITAL}

0 Arencibia Sanchez*, AF Rave Ramirez, D González García-Cano, M Laseca Modrego, A Martín Martínez. Complejo Hospitalario Universitario Insular Materno Infantil de Gran Canaria, Gynecologic Oncology, las palmas de gran canaria, Spain

\subsection{6/ijgc-2021-ESGO.56}

Introduction/Background* The objective is to know our degree of compliance with the ESGO 2019 quality indicators in surgical management of cervical cancer

Methodology Retrospective study of patients with cervical cancer who underwent laparoscopic radical hysterectomy in the 